\title{
Cuidados farmacêuticos e o uso de Medicamentos Isento de Prescrição (MIPs)
}

\author{
Pharmaceutical care and the use of Over-the-Counter Medications (MIPs)
}

Atención farmacéutica y uso de Medicamentos de Venta Libre (MIP)

Recebido: 09/09/2021 | Revisado: 16/09/2021 | Aceito: 26/09/2021 | Publicado: 27/09/2021

\author{
Pedro Henrique Damascena Guimarães \\ ORCID: https://orcid.org/0000-0003-0973-2921 \\ Faculdade Integrada Carajás, Brasil \\ E-mail: pedro_redex@hotmail.com \\ Rodrigo Pereira Pacheco \\ ORCID: https://orcid.org/0000-0003-3543-5867 \\ Faculdade Integrada Carajás, Brasil \\ E-mail: rodrigpacheco@ hotmail.com \\ Yolanda de Jesus Morais \\ ORCID: https://orcid.org/0000-0001-7105-8267 \\ Faculdade Integrada Carajás, Brasil \\ E-mail:Yolandamorais123@gmail.com
}

\begin{abstract}
Resumo
Introdução: Os Medicamentos Isentos de Prescrição (MIPs) foram aprovados pelas autoridades sanitárias para tratar sintomas menores ou autolimitados, sendo comercializados nos autos serviços nas farmácias/drogarias e dispensados sem a apresentação da prescrição, nesse cenário integra-se o cuidado farmacêutico, como uma ferramenta provedora de saúde e orientação quanto ao uso correto e discriminado dos MIPs, uma vez que esse artigo tem como objetivo sintetizar evidências sobre a importância do cuidado farmacêutico na utilização dos MIPs, visando à segurança do paciente. Métodos: Revisão Integrativa e síntese narrativa, tendo como questão norteadora a existência de evidências sobre o cuidado farmacêutico na utilização dos MIPs, apresentando como desfechos a diminuição do uso indiscriminado dos MIPs mediante as orientações inerentes ao cuidado farmacêutico e a redução dos agravos a saúde provocados pelo uso dos MIPs de maneira errônea. O recorte temporal foi de 2011 a 2021. Buscou-se estudos publicados nas bases Cochrane Library, Epistemonikos, Health Evidence, Biblioteca Virtual de Saúde e Google Scholar. Resultados e discussão: estudos demonstram que o uso indiscriminado dos MIPs é elevadíssimo, podendo ocasionar uma séria de agravos a saúde e destacam ainda que é indispensável as informações referentes ao uso correto dos MIPs, dentre os principais riscos ocasionados pelo uso indiscriminado dos MIPs destaca-se a sobrecarga causada ao fígado, rins e pulmões; infecções de pele e sangramentos estomacais.Conclusão: Há evidências de que o cuidado farmacêutico proporciona a melhoria da adesão a terapia medicamentosa e redução dos problemas relacionados ao uso indiscriminado dos MIPs, porém devido ao fácil acesso, esses medicamentos ainda são usados em larga escala.
\end{abstract}

Palavras-chave: Cuidado farmacêutico; MIPs; Uso indiscriminado.

\begin{abstract}
Introduction: Over-the-counter Medicines (MIPs) were approved by health authorities to treat minor or self-limited symptoms, being marketed in self-service pharmacies/drugstores and dispensed without the presentation of a prescription. In this scenario, pharmaceutical care is integrated as a health provider tool and guidance on the correct and discriminated use of PIMs, as this article aims to synthesize evidence on the importance of pharmaceutical care in the use of PIMs, aiming at patient safety. Methods: Integrative review and narrative synthesis, having as a guiding question the existence of evidence on pharmaceutical care in the use of PIMs, presenting as outcomes the reduction of the indiscriminate use of PIMs through the guidelines inherent to pharmaceutical care and the reduction of health problems caused by misusing MIPs. The time frame was from 2011 to 2021. Studies published in the Cochrane Library, Epistemonikos, Health Evidence, Virtual Health Library and Google Scholar databases were searched. Results and discussion: studies show that the indiscriminate use of MIPs is extremely high, which can cause serious health problems and also highlight that information regarding the correct use of MIPs is essential, among the main risks caused by the indiscriminate use of MIPs. if the overload caused to the liver, kidneys and lungs; skin infections and stomach bleeding. Conclusion: There is evidence that pharmaceutical care improves adherence to drug therapy and reduces problems related to the indiscriminate use of PIMs, but due to their easy access, these drugs are still used on a large scale.
\end{abstract}

Keywords: Pharmaceutical care; MIPs; Indiscriminate use. 


\begin{abstract}
Resumen
Introducción: Los medicamentos de venta libre (MIP) fueron aprobados por las autoridades sanitarias para el tratamiento de síntomas menores o autolimitados, se comercializan en farmacias / droguerías de autoservicio y se dispensan sin la presentación de receta médica. En este escenario, la atención farmacéutica se integra como una herramienta del proveedor de salud y una guía sobre el uso correcto y discriminado de los PIM, ya que este artículo tiene como objetivo sintetizar evidencia sobre la importancia de la atención farmacéutica en el uso de PIM, con miras a la seguridad del paciente. Métodos: Revisión integrativa y síntesis narrativa, teniendo como pregunta orientadora la existencia de evidencia sobre la atención farmacéutica en el uso de PIM, presentando los resultados de la reducción del uso indiscriminado de PIM a través de lo inherente a la atención farmacéutica y la reducción de problemas de salud. causado por el mal uso de MIP. El período de tiempo fue de 2011 a 2021. Se realizaron búsquedas en los estudios publicados en las bases de datos Cochrane Library, Epistemonikos, Health Evidence, Virtual Health Library y Google Scholar. Resultados y discusión: los estudios muestran que el uso indiscriminado de MIP es extremadamente alto, lo que puede ocasionar graves problemas de salud y también resaltan que la información sobre el correcto uso de los MIP es fundamental, entre los principales riesgos que genera el uso indiscriminado de MIP. si la sobrecarga causada en el hígado, los riñones y los pulmones; Infecciones cutáneas y sangrado de estómago Conclusión: Existe evidencia de que la atención farmacéutica mejora la adherencia a la farmacoterapia y los problemas relacionados con el uso indiscriminado de PIM, pero debido a su fácil acceso, estos fármacos se siguen utilizando a gran escala.
\end{abstract}

Palabras clave: Atención farmacéutica; MIP; Uso indiscriminado.

\title{
1. Introdução
}

Os Medicamentos Isentos de Prescrição (MIPs) foram aprovados pelas autoridades sanitárias para tratar sintomas menores ou autolimitados, sendo comercializados nos autos serviços nas farmácias/drogarias e dispensados sem a apresentação da prescrição (Santos; Bueno \& Muniz, 2020).

Os problemas de saúde autolimitados ou agravos menores são descritos como enfermidades agudas de baixa gravidade sendo corizas, dispepsias, dor de cabeça, tosse alérgica, cólicas, as principais (Miranda Filho; Andrade Júnior \& Montenegro, 2021).

A farmácia comunitária sempre é procurada pelos pacientes a fim de solucionar os problemas autolimitados, pois é o estabelecimento de saúde mais acessível para toda a população, gerando assim uma procura relativamente alta pelos (MIPs), nesse sentido é extremamente importante as orientações do profissional farmacêutico para que se possa obter uma terapia medicamentosa exitosa e segura, unificando dessa forma os cuidados com o paciente Miranda Filho; Andrade Júnior \& Montenegro, 2021).

A utilização indiscriminada dos MIPs favorece o crescimento dos problemas relacionados a medicamentos e das reações adversas. Essas reações adversas são descritas como um grave problema de saúde em todo o mundo, sendo responsáveis por diversas hospitalizações, pelo acréscimo do tempo de hospitalização e até mesmo por óbitos (Freitas et al., 2017).

Diante dos inúmeros problemas ocasionados pelo uso indiscriminado dos MIPs para tratar as doenças autolimitadas, essa pesquisa faz-se necessária a fim deevidenciar o impacto do cuidado farmacêutico no tocante a utilização dos MIPs.

Nesse cenário integra-se o cuidado farmacêutico, como uma ferramenta provedora de saúde e orientação quanto ao uso correto e discriminado dos MIPs, uma vez que esse artigo tem como objetivo sintetizar evidências sobre a importância do cuidado farmacêutico na utilização dos MIPs, visando à segurança do paciente.

\section{Conceito e definição dos MIPs}

No Brasil, somente os MIPs podem ser divulgados ao público leigo. De acordo com a OMS, os MIPs são medicamentos que tem autorização sanitária para tratar os problemas autolimitados, são dispensados sem a obrigatoriedade da prescrição médica, pois apresentam segurança e eficácia desde que sejam utilizados de acordo com as orientações descritas nas bulas e rótulos (Abimip, 2019). 
De modo geral são utilizados para tratar dores de cabeça, acidez estomacal, azia, febre, tosse, assaduras, prisão de ventre, aftas, dor de garganta, hemorroidas, congestão nasal (Soterio \& Santos, 2016).

No ano de 1973, os MIPs foram citados pela primeira vez através da legislação sanitária brasileira de $\mathrm{n}^{\circ} .5 .991$, de 17 de dezembro mencionados que apresenta como deve ser realizado o controle sanitário desses medicamentos. Posteriormente, em 2003, a Anvisa publicou a $\operatorname{RDC} \mathrm{n}^{\circ} .138$, de 29 de maio (republicada em 6 de janeiro de 2004), sendo o primeiro regulamento dos MIPs existente no país (Abimip, 2019).

De acordo com a ANVISA (2016), as características que o medicamento deve apresentar, para que o mesmo possa ser considerado MIP devem ser:

Utilizado para tratar patologias não graves e que apresente evolução lenta ou inexistente (ANVISA, 2016).

Apresentar reações adversas e possuir reações adversas com eventualidades conhecidas, além de apresentar baixo potencialde toxicidade e de interações medicamentosas (ANVISA, 2016).

Os MIPs devem ser usados por um breve período ou pelo tempo descrito na bula, quando o medicamento for de uso profilático, uma vez que não existe MIP de uso contínuo. Os MIPs precisam ser medicamentos que apresentem o mínimo risco potencial possível ao paciente e não apresentar dependência química e psíquica (Marinho \& Meirelles, 2021).

De acordo com a RDC nº. 98/2016, os MIPs são medicamentos disponíveis nos autos serviços em farmácias e drogarias, não tendo a necessidade de apresentação da prescrição médica para serem dispensados.

Outra característica dos MIPs é quanto a embalagem, pois as embalagens dos MIPs não apresentam tarja, como as embalagens dos medicamentos sujeitos a prescrição que são aqueles comercializados na tarja vermelha e também os medicamentos sujeitos a controle especial contidos na portaria 344/98 da Anvisa, que são comercializados na tarja preta (Brasil, 2016).

\section{Possíveis riscos decorrente da utilização inadequada dos MIPs}

Os analgésicos, antitérmicos e anti-inflamatórios são os medicamentos mais utilizados no Brasil, e alguns princípios ativos dessas classes fazem parte dos MIPs (Soterio \& Santos, 2016).

A fácil acessibilidade dos MIPs pela população favorece o uso e a finalidade incorreta do medicamento, o pode gerar danos à saúde como qualquer outro medicamento. O ácido acetilsalicílico, a dipirona e o paracetamol, são medicamentos utilizados em larga escala e estão mencionados na lista dos MIPs, porém quando esses medicamentos são usados de maneira errada, pode gerar danos gravíssimos para a saúde do paciente, uma vez que todos os medicamentos existentes no mercado farmacêutico apresentam efeitos adversos (Soterio \& Santos, 2016).

A utilização de Anti-inflamatórios não esteroides (AINEs) em idosos está diretamente relacionada ao elevado risco de toxicidade gastrointestinal e insuficiência renal, além de ter relação com eventos cardiovasculares e várias interações medicamentosas (O’Neil; Hanlon; Marcum, 2012).

Geralmente os anti-inflamatórios precisam ser evitados em pacientes idosos, especialmente naqueles que apresentam úlcera péptica, doença crônica no fígado, cardíacos ou hipertensão arterial e que usam anticoagulantes. Diversos estudos têm apontado que a prevalência de prescrições com medicamentos que apresentam interações entre si em pacientes que usam antiinflamatórios é elevada e preocupante (Ely et al., 2015).

Os MIPs são utilizados com bastante frequência, devido a impressão comum de baixo risco, entretanto dados da Fiocruz relatam que, para o ano de 2012, os episódios de intoxicação por medicamentos foram responsáveis por $21 \%$ das mortes no Brasil (Fiocruz, 2012).

Outro fator que contribui para o uso abusivo dos MIPs são as inúmeras publicidades de medicamentos conduzidas pela mídia são frequentemente elaboradas sem avaliar os critérios científicos, assinalando apenas os benefícios dos 
medicamentos, não levando em consideração os pontos negativos, induzindo de forma negativa o consumo, o uso indiscriminado e, por conseguinte, aumentando os casos de intoxicações medicamentosa (Alexandri et al., 2011).

\section{Importância do cuidado farmacêutico no uso correto dos MIPs}

Na década de 60 aconteceu um movimento chamado Farmácia Clínica realizado por acadêmicos e professores da Universidade de São Francisco (EUA), que teve como finalidade a aproximação do profissional farmacêutico com a equipe de saúde, tornando possível a realização de atividades inerentes a farmacoterapia em ambiente hospitalar (Barros; Silva; Leite, 2020).

Devido as mudanças demográficas e o aumento da morbimortalidade referente as doenças transmissíveis e não transmissíveis, a área da saúde exigiu novos perfis do profissional farmacêutico. Nesse sentido, o conceito do Pharmaceutical Care está sendo incluído nos diversos níveis de atenção à saúde desde a década de 70 (Barros; Silva; Leite, 2020).

Entende-se como cuidado farmacêutico, a prática profissional na provisão adequada e responsável da farmacoterapia, com o objetivo de atingir uma terapia farmacológica exitosa, que venha de fato proporcionar qualidade de vida ao paciente (CFF 2016).

Nesse contexto, o Conselho Federal e Farmácia (CFF), regulamentou por meio da Resolução de no 586 de 29 de agosto de 2013, a prescrição farmacêutica, com o intuito de favorecer a utilização correta dos medicamentos, principalmente quando se refere ao uso dos MIPs, uma vez, que são alvos da automedicação por estarem dispostos nos estabelecimentos farmacêuticos ao alcance do cliente, favorecendo o seu uso indiscriminado. Essa resolução, foi um marco significativamente positivo para a profissão farmacêutica, pois a mesma possibilitou uma maior visibilidade do profissional farmacêutico nas farmácias e drogarias comunitárias espalhadas, por todo o país (CFF, 2013).

Após a criação dessa resolução, o profissional farmacêutico pôde desenvolver estratégias mais concretas para minimizar o uso indiscriminado de medicamentos, como a elaboração da prescrição farmacêutica envolvendo na mesma o tratamento farmacológico e não farmacológico dentro de um plano de cuidado que é elaborado em comum acordo com o paciente observando o contexto real em que o mesmo vive, tendo como objetivo principal o estímulo ao uso racional e correto dos MIPs, proporcionando a este uma melhor qualidade de vida (CFF, 2013).

É válido destacar que o Cuidado Farmacêutico é centrado no paciente, desse modo o profissional passa a assumir a responsabilidade de cuidador do paciente, mediante o acompanhamento farmacoterapêutico que é realizado com o paciente após a elaboração do plano de cuidado, esse acompanhamento se faz necessário, uma vez, que o farmacêutico precisa avaliar os resultados das intervenções realizadas com o paciente (Lima; Silva; Guedes, 2020).

O profissional farmacêutico, deve investir na educação em saúde no seu estabelecimento de trabalho, seja ele público ou privado criando dessa forma um vínculo de confiança com o paciente de forma que favoreça o uso consciente e seguro dos MIPs, pois na medida que o paciente começa a entender os riscos do uso indiscriminado dos MIPs o mesmo passará a utilizar o medicamento de forma correta e quando realmente for necessário (Lima et al., 2018).

\section{Metodologia}

Trata-se de uma Revisão Integrativa (Cunha; Cunha; Alves, 2014), tendo como questão norteadora a existência de evidências sobre o cuidado farmacêutico na utilização dos MIPs, tendo como desfechos a diminuição do uso indiscriminado dos MIPs mediante as orientações inerentes ao cuidado farmacêutico e a redução dos agravos a saúde provocados pelo uso dos MIPs de maneira errônea. O recorte temporal foi de 2011 a 2021. Buscou-se estudos publicados nas bases Cochrane Library, Epistemonikos, Health Evidence, Biblioteca Virtual de Saúde e Google Scholar. Para a estratégia de busca, utilizou-se os descritores "pharmaceutical care and use of MIP's" e "MIP's and indiscriminate use of medications", adaptados ao Medical 
Subject Headings (MeSH) e Descritores em Ciências da Saúde (DeCS), associados com operadores boleanos "AND" e "OR". Restringiu-se a pesquisa as línguas portuguesa, espanhola e inglesa.

Utilizou-se como critérios de inclusão: População: Pacientes que utilizam os MIPs; Intervenção: Cuidado Farmacêutico; Controle: Não utilização do cuidado farmacêutico ou qualquer orientação farmacêutica; Desfecho: diminuição do uso indiscriminado dos MIPs mediante as orientações inerentes ao cuidado farmacêutico e a redução dos agravos a saúde provocados pelo uso dos MIPs de maneira errônea. Não foram avaliados a qualidade dos artigos selecionados. Foram recuperados 148 registros, sendo 2 da BVS, 1 da Cochrane, 8 do Epistemonikos, 3 do Health Evidence, 2 do NCBI, 132 do Google Scholar e 3 registros identificados por outras fontes. Foram removidas 50 duplicatas, sendo ao final selecionados 8 artigos (Figura 1). Os resultados estão apresentados por meio de síntese narrativa.

Figura 1 - Fluxograma da seleção dos artigos incluídos.
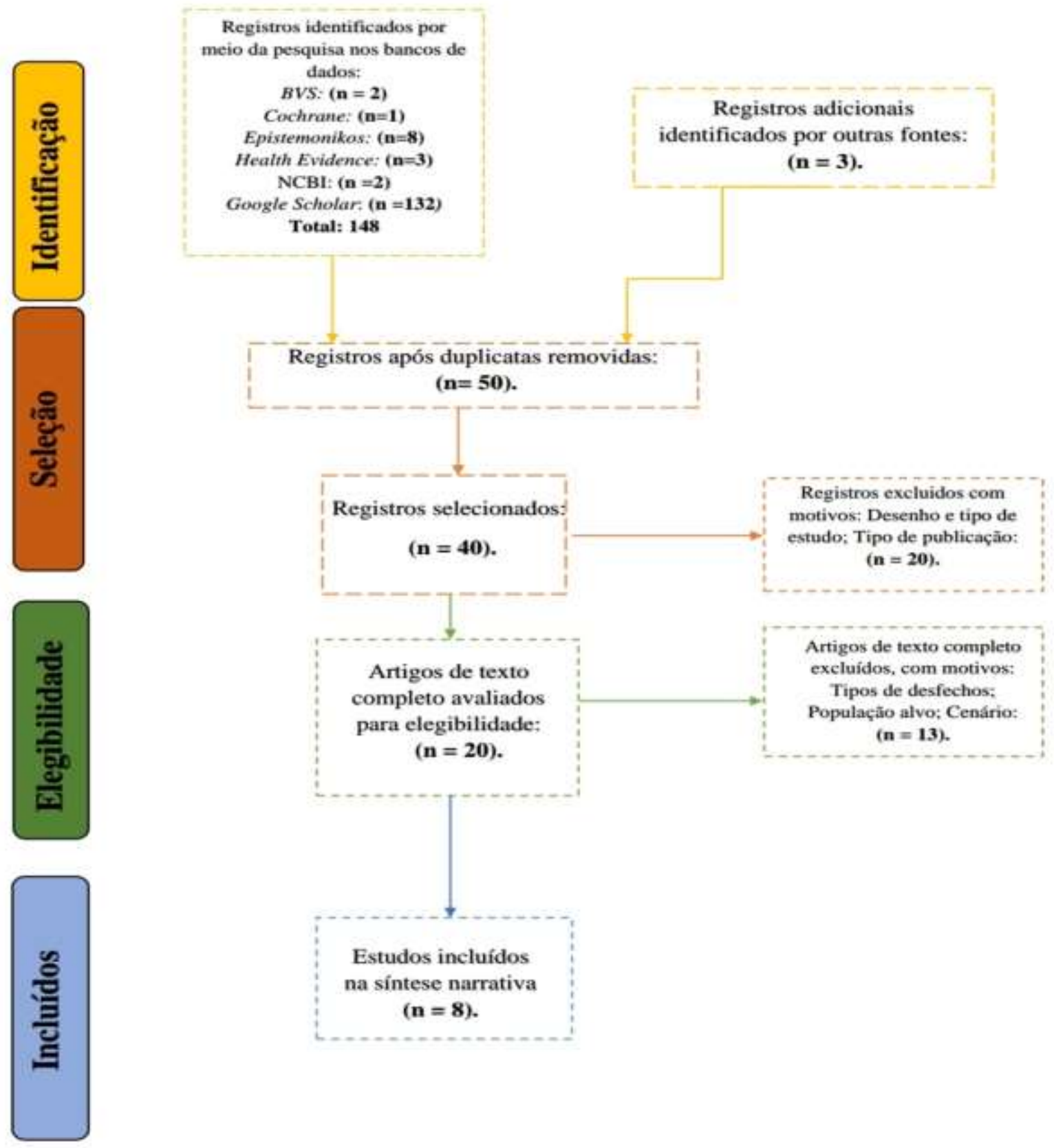

Fonte: Adaptado de Moher et al. (2009). 


\section{Resultados e Discussão}

8 artigos foram selecionados, sendo 1 de revisão integrativa, 2 de revisão de literatura e 5 estudos transversais. o perfil e características dos estudos estão apresentados na

tabela 1. os achados evidenciam que o uso do cuidado farmacêutico gera a redução dos problemas relacionados a medicamentos e uso indiscriminado de medicamentos.

Tabela 1 - Perfil e características dos artigos selecionados (Continua)

\begin{tabular}{|c|c|c|c|}
\hline ARTIGO & OBJETIVO & DESENHO DO ESTUDO & DESFECHO \\
\hline Marinho \& Meirelles., (2021). & $\begin{array}{l}\text { Identificar quais são os MIPs mais } \\
\text { dispensados e os riscos associados à sua } \\
\text { utilização indiscriminada. }\end{array}$ & Revisão Integrativa. & $\begin{array}{l}\text { O farmacêutico tem um importante papel } \\
\text { como profissional que pode colaborar com } \\
\text { a instrução do paciente, orientando-o na } \\
\text { seleção da melhor opção terapêutica de } \\
\text { modo individualizado, a partir de uma } \\
\text { anamnese prévia, bem como promovendo o } \\
\text { uso racional de medicamentos e alertando } \\
\text { os consumidores sobre os principais riscos } \\
\text { advindos da automedicação. }\end{array}$ \\
\hline Oliveira et al., (2020). & $\begin{array}{l}\text { Realizar um levantamento do uso de } \\
\text { medicamentos isentos de prescrição em } \\
\text { acadêmicos dos cursos de farmácia e de } \\
\text { medicina em uma instituição privada de } \\
\text { ensino superior em São Paulo/SP. }\end{array}$ & Descritivo Transversal. & $\begin{array}{l}\text { O estudo reforça a indispensabilidade da } \\
\text { conscientização do uso correto de } \\
\text { medicamentos no ambiente acadêmico, } \\
\text { evitando que essa prática desenfreada seja } \\
\text { passada para a população. }\end{array}$ \\
\hline Magalhães et al., (2020). & $\begin{array}{l}\text { Identificar o conhecimento dos pacientes } \\
\text { atendidos em uma Unidade de Atenção } \\
\text { Primária à Saúde sobre o uso de } \\
\text { Medicamentos Isentos de Prescrição }\end{array}$ & Descritivo Transversal. & $\begin{array}{l}\text { Ainda existe muita desinformação a } \\
\text { respeito dos MIPs, tanto em relação à sua } \\
\text { definição quanto aos seus riscos. Ademais, } \\
\text { notou-se que poucos têm o hábito de se } \\
\text { consultar com o farmacêutico que é o } \\
\text { profissional de saúde que apresenta maior } \\
\text { grau de conhecimento sobre esses } \\
\text { medicamentos. Nesse sentido salienta-se } \\
\text { que o cuidado farmacêutico é uma prática } \\
\text { que reduz os riscos e o uso inapropriado } \\
\text { dos MIPs. }\end{array}$ \\
\hline
\end{tabular}


Research, Society and Development, v. 10, n. 12, e485101220405, 2021

(CC BY 4.0) | ISSN 2525-3409 | DOI: http://dx.doi.org/10.33448/rsd-v10i12.20405

Santos; Bueno \& Muniz., (2020).

Fernandes \& Cembraneli (2014).

Freitas el al., (2017)

Piecuch; Makarewicz-Wujec \& Kozłowska-Wojciechowska., (2017).

Rutter \& Wadesango., (2014).
Criação e teste de um aplicativo móvel, que permite ao usuário ter acesso a um bulário intuitivo e acessível, com informações diretas e claras a respeito de MIP.
Estudo Transversal.

Enfatizar questões sobre a automedicação, o uso irracional de MIPs e, ainda, demonstrar a importância do profissional farmacêutico no combate a essas práticas.

Identificar, dentre os MIPs, os mais vendidos e causadores de intoxicações, destacando o risco tóxico do paracetamol, isoladamente ou em associação com outros fármacos.

Explorar a interação entre construtos
selecionados (farmacêutico centrado no paciente e competência) que podem influenciar o fornecimento de informações sobre medicamentos pelos farmacêuticos. O estudo foi realizado na perspectiva de um farmacêutico comunitário.

Explorar como os farmacêuticos comunitários usam evidências para informar sua prática ao recomendar ou vender medicamentos sem prescrição.

Revisão de Literatura
MediVoz pode ser uma ferramenta prática e acessível no acesso a informações referente aos MIP, não substituindo o profissional de saúde capacitado, porém auxiliando e melhorando o entendimento, principalmente por contar com a pronúncia automática das informações.

A prescrição farmacêutica apresenta-se como ferramenta de segurança, garantindo aos pacientes o uso correto de medicamentos, sendo o principal beneficiado da atuação farmacêutica a população brasileira.

Revisão de Literatura. $\quad \begin{aligned} & \text { medicamentos, sendo o principal } \\ & \text { benaça farmacêtica a }\end{aligned}$

O farmacêutico desempenha relevante papel na monitorização e prevenção de intoxicações por paracetamol.

O cuidado farmacêutico é centrado no paciente e considera o bem-estar do paciente, nesse sentido existe uma maior possibilidade de que o farmacêutico forneça informações sobre o uso correto dos medicamentos MIPs.

As orientações do profissional farmacêutico facilita a adesão á terapia medicamentosa e diminui o uso indiscriminado de medicamentos.

Fonte: Cuidado farmacêutico e o uso dos MIPs (2021). 
Research, Society and Development, v. 10, n. 12, e485101220405, 2021

(CC BY 4.0) | ISSN 2525-3409 | DOI: http://dx.doi.org/10.33448/rsd-v10i12.20405

Tabela 2: Desfechos apresentados sobre o Cuidado Farmacêutico e o uso de MIPs.

\begin{tabular}{|c|c|c|c|}
\hline \multirow[t]{2}{*}{ ARTIGO } & \multicolumn{3}{|c|}{ DESFECHOS } \\
\hline & $\begin{array}{l}\text { Melhoria da resolução de problemas } \\
\text { relacionados com o uso dos MIPs }\end{array}$ & $\begin{array}{l}\text { Uso do cuidado } \\
\text { farmacêutico }\end{array}$ & Utilização indiscriminada dos MIPs. \\
\hline Marinho \& Meirelles., (2021). & & & $(+)$ \\
\hline Oliveira et al., (2020). & & $(+)$ & $(+)$ \\
\hline Magalhães et al., (2020). & & $(+)$ & $(+)$ \\
\hline Santos; Bueno \& Muniz., (2020). & $(+)$ & $(+)$ & \\
\hline Fernandes \& Cembraneli (2014). & & $(+)$ & $(+)$ \\
\hline Freitas et al., (2017). & & & $(+)$ \\
\hline $\begin{array}{l}\text { Piecuch; Makarewicz-Wujec \& } \\
\text { Kozłowska-Wojciechowska., (2017). }\end{array}$ & $(+)$ & $(+)$ & \\
\hline Rutter \& Wadesango., (2014). & $(+)$ & $(+)$ & \\
\hline
\end{tabular}

Fonte: Cuidado farmacêutico e o uso dos MIPs (2021). 
Marinho e Meirelles (2021), constataram que o emprego indiscriminado dos MIPs pode ocasionar inúmeros riscos à saúde. Em meio aos principais riscos do uso inadequado destaca-se a sobrecarga causada ao fígado, rins e pulmões; infecções de pele e sangramentos estomacais. Nesse contexto é destacado a importância do profissional farmacêutico no fornecimento de informações necessários, com o intuito de alertar os consumidores dos riscos referentes a automedicação.

Oliveira et al., (2020) registraram que o uso indiscriminado dos MIPs na população estudada por eles é elevadíssimo, podendo ocasionar uma séria de agravos a saúde dos mesmos, destacam ainda que é indispensável as informações referentes ao uso correto dos MIPs por parte do profissional farmacêutico, a fim de gerar a diminuição dessa prática indiscriminada.

Magalhães et al., (2020) demonstram que existe uma grande desinformação a respeito do uso e definição dos MIPs por grande parte da população, salientando que a prática do cuidado farmacêutico é uma técnica que pode auxiliar na diminuição dos problemas gerados pela utilização indiscriminada desses medicamentos.

Santos; Bueno e Muniz., (2020), constataram que o aplicativo Medivoz pode ser empregado como uma ferramenta que favorece as informações inerentes ao uso dos MIPs, porém esse aplicativo não substitui as orientações realizadas pelo profissional farmacêutico no ato da dispensação.

Fernandes e Cembraneli (2014), inferem que o cuidado farmacêutico se configura como uma estratégia de segurança, assegurando aos pacientes o uso adequado de medicamentos, tendo o paciente como o principal beneficiado.

Freitas et al., (2017), constataram que os MIPs formam uma expressiva parcela econômica na indústria e também no varejo farmacêutico tendo um consumo em constante crescimento em todo o mundo, nesse sentido existe a necessidade de estimular o cuidado farmacêutico devido os riscos tóxicos e o aumento da automedicação.

Piecuch; Makarewicz-Wujec e Kozłowska-Wojciechowska., (2017), demonstram que o profissional farmacêutico é capacitado para desempenhar ações inerentes ao cuidado farmacêutico o que possibilita que o mesmo desenvolva ações que minimizem os problemas relacionados a medicamentos e o uso indiscriminado do mesmo, favorecendo a saúde e bem-estar do paciente.

Rutter e Wadesango., (2014), demonstram que o cuidado farmacêutico facilita a adesão a terapia medicamentosa e busca minimizar os danos ocasionados pelo uso indiscriminado.

\section{Conclusão}

Há evidências de que o cuidado farmacêutico proporciona a melhoria na adesão a terapia medicamentosa e redução dos problemas relacionados ao uso indiscriminado dos MIPs, porém devido ao fácil acesso, esses medicamentos ainda são usados em larga escala.

\section{Implicações práticas}

Recomenda-se a necessidade de estudos complementares quanto ao emprego do cuidado farmacêutico como ferramenta facilitadora da adesão a terapia medicamentosa, diminuição do uso indiscriminado e redução dos problemas relacionados a medicamentos.

\section{Contribuições dos autores}

RPP e PHDG concebeu a estratégia de pesquisa em conjunto com YJM. RPP e PHDG fez as buscas bibliográficas nas bases de dados, a seleção dos artigos e a extração de dados sob a supervisão de YJM. Os autores leram e aprovaram a versão final do manuscrito. O conteúdo da revisão é de exclusiva responsabilidade individuais dos autores. 
Research, Society and Development, v. 10, n. 12, e485101220405, 2021

(CC BY 4.0) | ISSN 2525-3409 | DOI: http://dx.doi.org/10.33448/rsd-v10i12.20405

\section{Declaração de conflito de interesses}

Os autores declaram que a pesquisa foi conduzida na ausência de quaisquer relações comerciais ou financeiras que possam ser interpretados como um potencial conflito de interesse.

\section{Referências}

Abimip - Associação Brasileira da Indústria de Medicamentos Isentos dePrescrição. Conheça o MIP, 2019 https://abimip.org.br/texto/conhecao-mip .

Alexandri, M., Foppa, A. A., Welter, A. C., Campos, C. M. T., Cunha, H. P., Scherer, M. L. S., \& Zannin, M. (2011). Propaganda de medicamentos: um desafio para todas as profissões. Revista Brasileira de Farmácia.

Barros, D. S. L., Silva, D. L. M., \& Leite, S. N. (2019). Serviços farmacêuticos clínicos na atenção primária à saúde do Brasil. Trabalho, Educação e Saúde, 18.

Brasil. Agência Nacional de Vigilância Sanitária - ANVISA. Resolução de Diretoria Colegiada. № 96 , de 17 de dezembro de (2008) Dispõe sobre a propaganda, publicidade, informação e outras práticas cujo objetivo seja a divulgação ou promoção comercial de medicamentos.http:// www.anvisa.gov.br/propaganda/rdc/rdc_96_2008_consolidada

Cff (Conselho Federal de Farmácia). Resolução Nº 586 de 29 de agosto de (2013). Regula a prescrição farmacêutica e dá outras providências. Brasília. Distrito Federal.

Cunha, P. L. P., Cunha, C. S., Alves, P. F. (2014) Revisão bibliográfica sistemática integrativa: a pesquisa baseada em evidências. Revista Anima Educação. 6(3):1-63. http://dx.doi.org/10.1590/ S0103-21002007000200001.

da Silva, G. C., de Oliveira, J. B., de Freitas, V. D. M., \& de Oliveira, D. F. (2020). Levantamento do uso de Medicamentos Isentos de Prescrição em acadêmicos dos Cursos de Farmácia e de Medicina em uma Instituição Privada de Ensino Superior em São Paulo/SP. Revista Brasileira de Ciências Biomédicas, 1(2), 72-77.

de Farmácia, C. F. (2016). Serviços farmacêuticos diretamente destinados ao paciente, à família e à comunidade: contextualização e arcabouço conceitual. Brasília: Conselho Federal de Farmácia, 200.

de Miranda Filho, J. P., de Andrade Júnior, F. P., \& de Albuquerque Montenegro, C. (2021). Cuidados farmacêuticos e os medicamentos isentos de prescrição: revisão integrativa da literatura. Archives of Health Investigation, 10(1), 153-162:http://dx.doi.org/10.21270/archi.v10i1.4903 .

de Sousa Marinho, L. N., \& Meirelles, L. M. A. (2021). Os riscos associados ao uso de medicamentos isentos de prescrição. Revista Saúde Multidisciplinar, $9(1)$.

dos Santos, M. J. R., Bueno, M., \& Muniz, J. J. (2020). MediVoz: Aplicativo de auxílio ao uso de medicamentos isentos de prescrição. Infarma-Ciências Farmacêuticas, 32(3), 292-299.

Ely, L. S., Engroff, P., Guiselli, S. R., Cardoso, G. C., Morrone, F. B., \& Carli, G. A. D. (2015). Uso de anti-inflamatórios e analgésicos por uma população de idosos atendida na Estratégia Saúde da Família. Revista Brasileira de Geriatria e Gerontologia, 18, 475-485: https://www.scielo.br/pdf/rbgg/v18n3/18099823-rbgg-18-03-00475.pdf .

Fernandes, W. S., \& Cembranelli, J. C. (2015). Automedicação e o uso irracional de medicamentos: o papel do profissional farmacêutico no combate a essas práticas. Revista Univap, 21(37), 5-12.

Freitas, J. A. B., de França Fonteles, M. M., de Sousa Lima, M. E., Bachur, T. P. R., \& Carvalho, T. M. D. J. P. (2017). Medicamentos isentos de prescrição: perfil de consumo e os riscos tóxicos do paracetamol. Revinter, 10(3), 134-154.

Magalhães, J. L. M., Santos, S. A., Belém, B. L. M., de Sousa, I. N. A., Maciel, D. A., Maia, I. D. F. V. C., \& Romero, N. R. (2021). Avaliação do conhecimento de pacientes de uma unidade de atenção primária à saúde acerca de medicamentos isentos de prescrição. Brazilian Journal of Health Review, 4(2), 6485-6501.

Moher, D., Liberati, A., Tetzlaff, J. \& Altman, D.G. (2009). The PRISMA Group. Preferred Reporting Items for Systematic Reviews and Meta-Analyses: The

Piecuch, A., Makarewicz-Wujec, M., \& Kozłowska-Wojciechowska, M. (2017). Improving the provision of OTC medication information in community pharmacies in Poland. International journal of clinical pharmacy, 39(1), 70-77.

Rutter, P., \& Wadesango, E. (2014). Does evidence drive pharmacist over-the-counter product recommendations?. Journal of evaluation in clinical practice, 20(4), 425-428.

Sinitox. (2012). Sistema Nacional de Informações Tóxico-Farmacológicas. Fundação Oswaldo Cruz.

Soterio, K. A., \& dos Santos, M. A. (2016). A automedicação no Brasil e a importância do farmacêutico na orientação do uso racional de medicamentos de venda livre: uma revisão. Revista da Graduação, 9(2):https://repositorio.pucrs.br/dspace/handle/10923/12308 . 